\title{
ANÁLISIS NO LINEAL DE MALLAS ESPACIALES DE DOBLE CAPA
}

\author{
(NON-LINEAR ANALYSIS OF DOUBLE-LAYER \\ SPACE GRIDS)
}

Fco. Javier Estévez Cimadevila, Dr. Arquitecto Profesor Cálculo de Estructuras E.T.S. Arquitectura. LA CORUÑA ESPAÑA

\section{RESUMEN \\ Este artículo corresponde al tema de investigación desarrollado en mi tesis doctoral.}

Se plantea un nuevo modelo para describir el comportamiento no lineal de piezas rectas biarticuladas sometidas a compresión, basado en la obtención de las ecuaciones constitutivas en el caso de los perfiles tubulares huecos de sección circular. Se obtienen las funciones de estabilidad de barras imperfectas y la formulación analítica del comportamiento postpandeo tomando la expresión exacta de la curvatura en la integración de la ecuación diferencial de la elástica.

La linearización de las leyes constitutivas y la aplicación de un modelo incremental iterativo basado en la modificación del módulo de elasticidad de las barras en estado crítico permite abordar el análisis no lineal de las mallas espaciales de doble capa.

Los modelos de análisis propuestos se aplicaron a una amplia variedad de mallas de diversas tipologías $y$ características de las que se acompañan algunos ejemplos en el artículo.

\section{SUMMARY}

This paper is related to the topic developed in my P.H.D. thesis.

A new model is proposed to describe the non-linear behaviour of straight pin-jointed compression members. Such a method is based upon obtaining the complete constitutive equations in the case of circular hollow sections. The stability functions of imperfect bars and the analytical formulation of the post-buckling behaviour are obtained by taking the exact expression of the curvature in the integration of the differential equation of the elastic.

The linearization of the constitutive relationships and the application of an incremental iterative model based upon the modification of the elasticity of the bars at critical point allows us to consider the non-linear analysis of the doublelayer space grids.

The models of analysis proposed here have been applied to a wide variety of trusses with different characteristics and typologies, some examples of which accompany this paper.

\section{INTRODUCCIÓN}

El dimensionado de una estructura espacial precisa de la consideración de dos estados límites:

ESTADO LÍMITE DE SERVICIO O UTILIZACIÓN para el que es válido en general, en estructuras de doble capa, un análisis lineal donde las deformaciones y esfuerzos son funciones lineales de las cargas aplicadas.

ESTADO LÍMITE ÚLTIMO DE AGOTAMIENTO, bien resistente, de inestabilidad por pandeo o porque la es. tructura se convierte en un mecanismo. En este caso, se precisa un análisis no lineal de la estructura como consecuencia de que tenemos que considerar dos tipos de efectos no lineales que afectan a su comportamiento:

- NO LINEALIDAD RESISTENTE, que englobaría la no linealidad mecánica y geométrica de las barras que conforman la malla una vez superado el límite de proporcionalidad del material que las constituye.

- NO LINEALIDAD GEOMÉTRICA DE LA ESTRUCTU. RA, cuando los desplazamientos de los nudos de la estructura son lo suficientemente importantes como para invalidar el planteamiento de las condiciones de equilibrio sobre la geometría no deformada. 
La consideración de estos efectos no lineales nos obliga, en un primer momento, al estudio de las relaciones constitutivas de las barras de la malla y, más específicamente, al estudio del PANDEO DE BARRAS BIARTICULADAS constituidas por perfiles tubulares de sección circular por ser las de utilización más frecuente en esta tipología estructural.

\section{ECUACIONES CONSTITUTIVAS DE LOS PERFILES TUBULARES HUECOS DE SECCION CIRCULAR}

\section{BARRAS PERFECTAS}

Se ha planteado el estudio del pandeo de barras biarticuladas en el tramo no proporcional de la ley tensión deformación.

La aplicación de la teoría del módulo tangente nos da Ia TENSION CRITICA REDUCIDA O COEFICIENTE DE REDUCCIÓN AL PANDEO, cuyo valor no depende de la forma de la sección y constituye el límite inferior de la resistencia máxima de una barra comprimida o instante en el cual se inicia la flexión lateral de la barra. Dicho valor viene dado por:

\section{(1)}

$$
\bar{N}=K_{1}+\sqrt{K_{1}^{2}-2 \cdot \bar{\sigma}_{p}-1}
$$

siendo $\mathrm{K}_{1}$ :

$$
K_{1}=\bar{\sigma}_{p}-\frac{1-\bar{\sigma}_{p}^{2}}{2 \bar{\sigma}_{\text {ouler }}}
$$

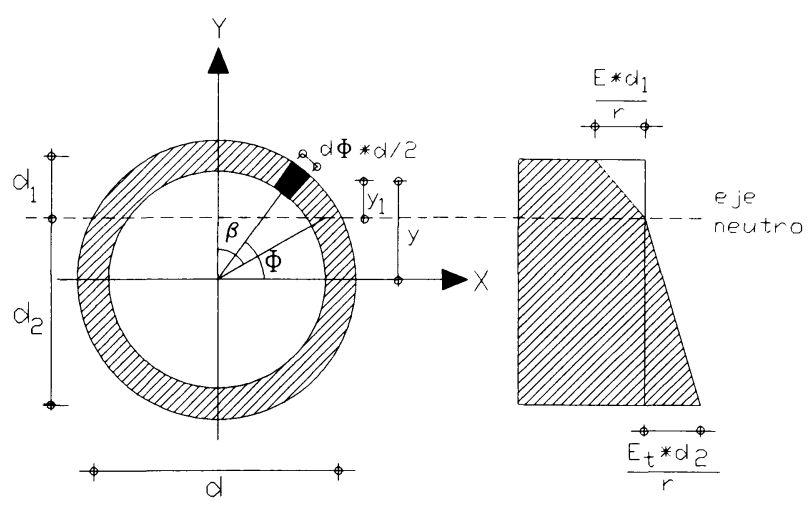

Fig. 1

Por otra parte, la teoría del módulo reducido o módulo doble, donde se plantea un módulo tangente para los incrementos de carga y el módulo de Young para los decrementos y que constituye la solución teórica correcta del pandeo en el intervalo no proporcional si consiguiésemos mantener la barra recta con la ayuda de restricciones exteriores hasta alcanzar el valor de carga crítica (es decir, constituye el límite superior de la resistencia máxima de una barra comprimida), depende de la forma de la sección por lo que fue precisa su aplicación a los perfiles tubulares huecos de sección circular con objeto de obtener la expresión del coeficiente de reducción al pandeo. Este coeficiente se obtierie a partir de la intersección de las ecuaciones (2) y (3) obtenidas, la primera a partir del planteamiento del equilibrio de tensiones en la sección transversal de la barra (Fig. 1) y la segunda por aplicación de la teoría de Euler donde el módulo de elasticidad se sustituye por el módulo reducido obtenido a partir del momento de flexión:

\section{[2]}

$$
\bar{N}=K_{4}+\sqrt{K_{4}^{2}-\bar{\sigma}_{p}+\frac{\left(1-\bar{\sigma}_{p}\right)^{2}}{K_{2}}}
$$

con

$$
K_{2}=1-\frac{1}{\pi} \cdot\left[\beta-\frac{3 \cdot \operatorname{sen} 2 \beta}{2 \cdot\left(1+2 \cdot \cos ^{2} \beta\right)}\right]
$$

$$
K_{3}=\sigma_{p}-\frac{\lambda^{2}}{2 \pi^{2} \cdot E} \cdot \frac{\left(\sigma_{f}-\sigma_{p}\right)^{2}}{K_{2}}
$$

$$
K_{1}=\bar{\sigma}_{p}-\frac{\left(1-\bar{\sigma}_{p}\right)^{2}}{2 \cdot K_{2} \cdot \bar{\sigma}_{\text {ouler }}}
$$

\section{[3]}

$$
N=\bar{\sigma}_{p}+\left(1-\bar{\sigma}_{p}\right) \cdot \sqrt{\frac{\pi}{\operatorname{tg} \beta-\beta+\pi}}
$$

En definitiva, con las ecuaciones (1), (2) y (3) tenemos relacionada la TENSIÓN CRITICA POR PANDEO con la esbeltez de la pieza, con lo que estamos en condiciones de abordar el problema del pandeo en barras imperfectas con el objetivo de conseguir la formulación de las funciones de estabilidad para su empleo en el análisis no lineal de mallas espaciales.

\section{BARRAS IMPERFECTAS}

Las piezas empleadas en la realidad no son piezas ideales ni perfectas tanto desde un punto de vista geométrico como desde un punto de vista físico o mecá- 
nico, y la existencia de un cierto grado de imperfecciones es la causa de que las tensiones críticas de pandeo no coincidan con las formulaciones teóricas desarrolladas para el caso de barras perfectas.

Frecuentemente las imperfecciones se engloban en dos grupos:

A) IMPERFECCIONES GEOMÉTRICAS.-Este primer grupo comprende las variaciones en las dimensiones de las piezas, su falta de rectitud y la inevitable excentricidad en la aplicación de la solicitación axil. Habitualmente se engloban en una combadura inicial.

B) IMPERFECCIONES ESTRUCTURALES.-Comprenderían la falta de homogeneidad del material, la existencia de tensiones residuales como consecuencia del propio proceso de fabricación, así como las variaciones del límite elástico del material sobre la sección de la barra.

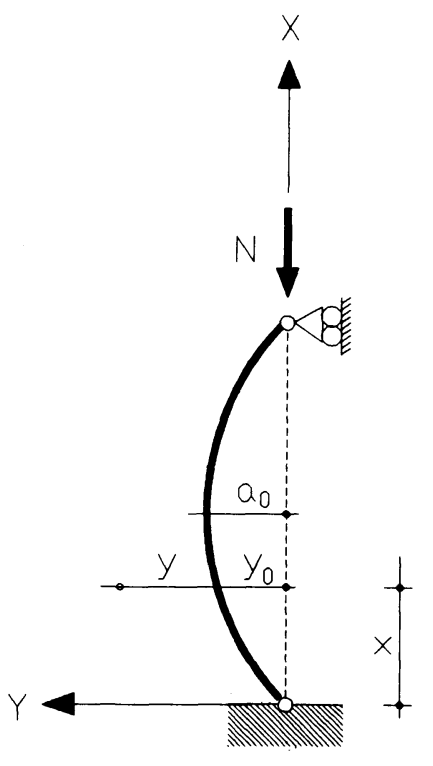

Fig. 2

Adoptando el criterio establecido por la Convención Europea de la Construcción Metálica (C.E.C.M.) de representar las imperfecciones en una combadura inicial y en una reducción del límite de elasticidad según el proceso de fabricación de los perfiles, podemos plantearnos el estudio analítico de la formulación de las funciones de estabilidad, tanto para el intervalo proporcional como no proporcional de la ley constitutiva del material de los perfiles, a partir del momento que origina la excentricidad de carga a lo largo del eje curvado de la pieza, lo que nos conduce a:
Función de estabilidad del pandeo elástico (Fig 3):

$$
\varepsilon=\frac{\sigma}{E}+\left[\frac{\pi}{F_{1} \cdot\left(1-\bar{\sigma} \cdot \lambda^{2}\right)}\right]^{2}
$$

con $F_{i}=L / a_{0}$, que constituye el factor de imperfección considerado.

Función de estabilidad del pandeo inelástico con aplicación de la teoria del módulo tangente (Fig. 4):

(5)

$$
\varepsilon=\frac{\sigma}{E \cdot\left[1-\left(\frac{\bar{\sigma}-\bar{\sigma}_{p}}{1-\bar{\sigma}_{p}}\right)^{2}\right]}+\frac{1}{4} \cdot\left[\frac{\pi}{F_{i} \cdot\left[1-\frac{\bar{\sigma} \cdot \bar{\lambda}^{2}}{1-\left(\frac{\bar{\sigma}-\bar{\sigma}_{p}}{1-\bar{\sigma}_{p}}\right)^{2}}\right]}\right]^{2}
$$

Función de estabilidad del pandeo inelástico con aplicación de la teoría del módulo reducido:

(6)

$$
\varepsilon=\frac{\sigma}{E \cdot\left[1-K_{2} \cdot\left(\frac{\bar{\sigma}-\bar{\sigma}_{p}}{1-\bar{\sigma}_{p}}\right)^{2}\right.}+\frac{1}{4} \cdot\left[\frac{\pi}{F_{i} \cdot\left[1-\frac{\bar{\sigma} \cdot \bar{\lambda}^{2}}{1-K_{2} \cdot\left(\frac{\bar{\sigma}-\bar{\sigma}_{p}}{1-\bar{\sigma}_{p}}\right)^{2}}\right]}\right]^{2}
$$

\section{COMPORTAMIENTO POSTPANDEO}

Ahora bien, dado que a nosotros nos interesa especialmente la colaboración de las barras pandeadas en la malla, es preciso el estudio teórico del postpandeo con objeto de alcanzar la formulación completa de la ecuación constitutiva.

La integración de la ecuación diferencial de la elástica obtenida con el valor exacto de la curvatura nos permite llegar a obtener la expresión del acortamiento por flexión originada por el pandeo en función del ángulo $\alpha$ que forma la tangente a la deformada en las articulaciones de los extremos con el eje rectilíneo de la ba. rra (Fig. 5).

\section{(7)}

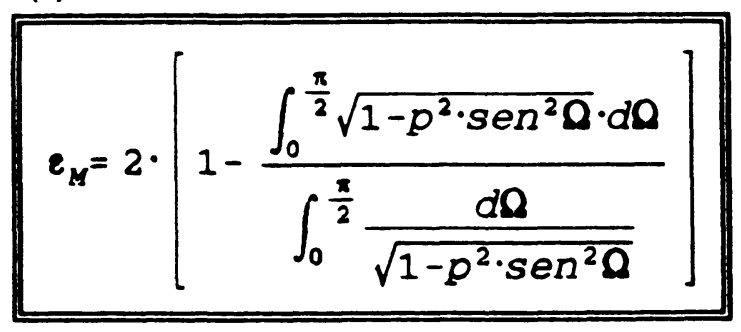

que converge por ser integrales elípticas de primera y segunda especie (Fig. 6). 


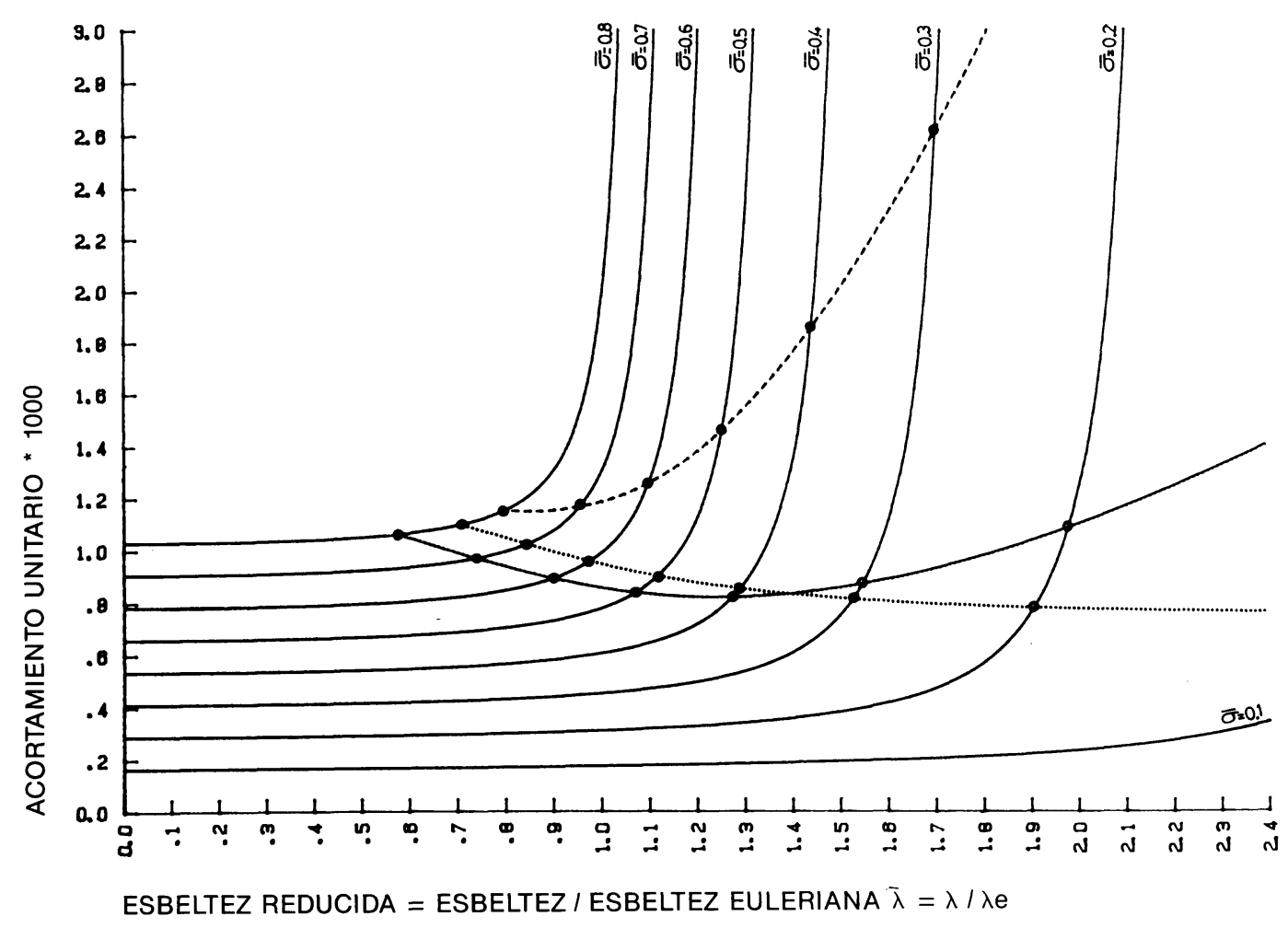

Fig. 3. PANDEO ELÁSTICO EN BARRAS IMPERFECTAS

Teoría de la preflecha convencional de Dutheil ...

Fórmula de Rondal y Maquoi para perfiles acabados en frío

Fórmula de Rondal y Maquoi para perfiles acabados en caliente _-_-_-_

Imperfección L/500 Acero A42.

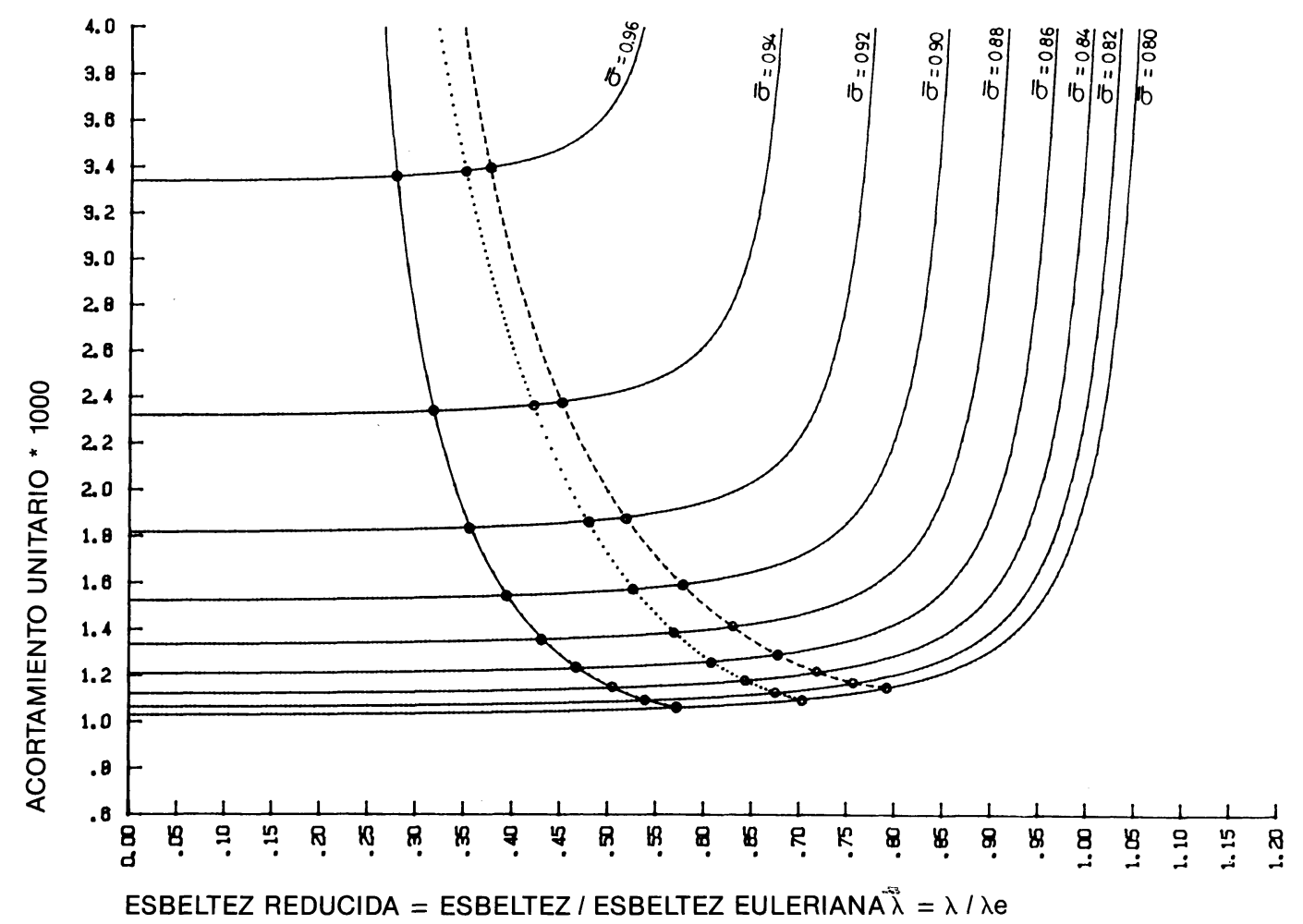

FIg. 4. PANDEO INELÁSTICO EN BARRAS IMPERFECTAS. MÓDULO TANGENTE

Teoría de la preflecha convencional de Dutheil

Fórmula de Rondal y Maquoi para perfiles acabados en frío

Fórmula de Rondal y Maquoi para perfiles acabados en caliente

Imperfección L/500 Acero A42. 


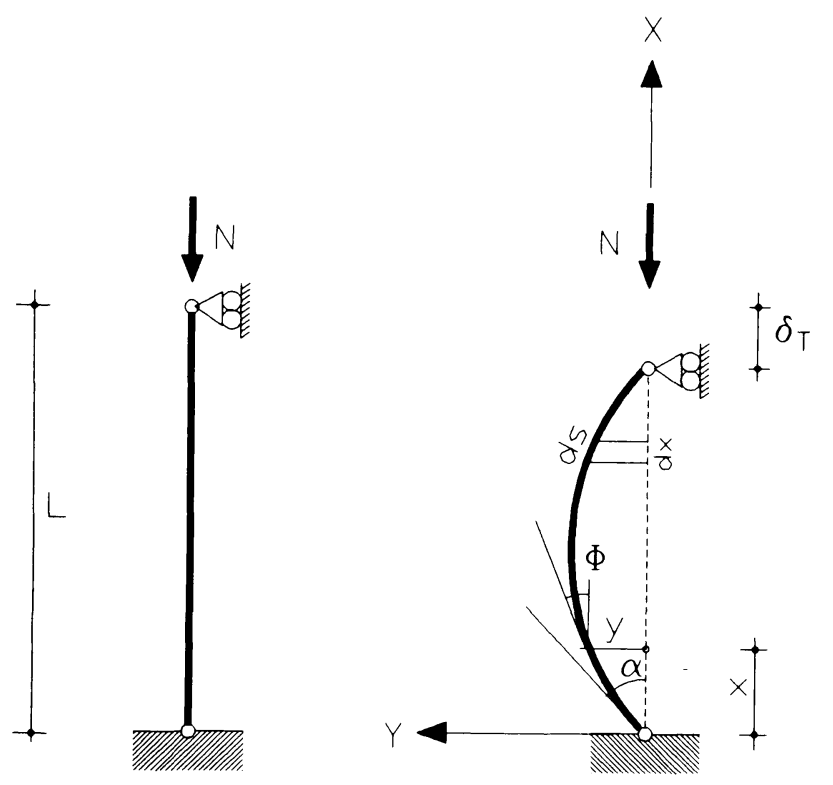

Fig. 5

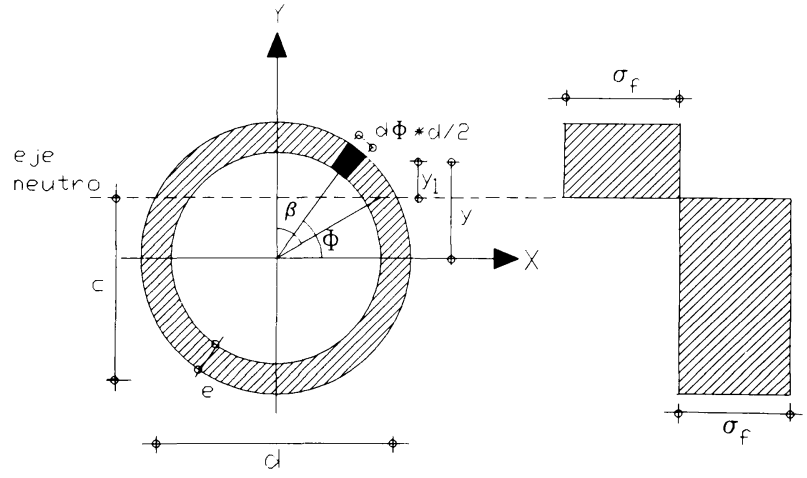

Fig. 6

El valor del ángulo $\alpha$ lo podemos obtener planteando el equilibrio de la sección transversal correspondiente al punto medio de la barra tomando el diagrama de tensiones de la Fig. 6, en la que hemos supuesto la sección totalmente plastificada, lo que nos permitirá determinar el valor de la flecha máxima que por equilibrio de la barra se corresponde con dicho diagrama y que iguala el valor de la flecha máxima de la elástica.

(8)

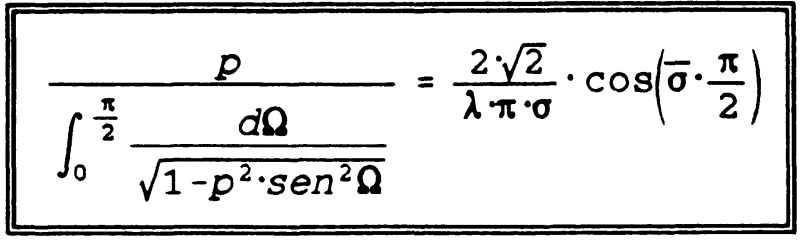

(c) Consejo Superior de Investigaciones Científicas Licencia Creative Commons 3.0 España (by-nc)
Por tanto, con la ecuación (8) se determina el valor de $\alpha$ en función del valor de la tensión reducida en el postpandeo.

El acortamiento debido al axil puede calcularse con:

Teoría del módulo tangente

(9)

$$
\varepsilon_{A}=\frac{\sigma_{f}}{E} \cdot\left[\bar{N} \cdot \frac{\left.(\bar{N}-\bar{\sigma})_{p}\right)^{2}}{\left(1-\bar{\sigma}_{p}\right)^{2}-\left(\bar{N}-\bar{\sigma}_{p}\right)^{2}}+\bar{\sigma}\right]
$$

Teoría del módulo reducido

(10)

$\varepsilon_{A}=\frac{\sigma_{E}}{E} \cdot\left[\bar{N} \cdot \frac{\left(\bar{N}-\bar{\sigma}_{p}\right)^{2} \cdot K_{3}}{\left(1-\bar{\sigma}_{p}\right)^{2}-\left(\bar{N}-\bar{\sigma}_{p}\right)^{2} \cdot K_{3}}+\bar{\sigma}\right]$

En definitiva, en el postpandeo, para un valor dado de tensión, el acortamiento unitario lo podemos obtener como suma del acortamiento axil y el acortamiento debido a la flexión por pandeo, empleando para ello las ecuaciones (7), (8), (9) y (10), RELACIONES CONSTITUTIVAS COMPLETAS de barras biarticuladas constituidas por perfiles tubulares huecos de sección circular con lo que estamos en condiciones de abordar el análisis no lineal de mallas espaciales.

En la Fig. 7 se han grafiado las referidas relaciones constitutivas para acero $\mathrm{A52}$, diferentes parámetros de esbeltez y una imperfección de L/500. Se puede observar como dichas relaciones reproducen tanto el pandeo elástico característico de barras de elevada esbeltez (con una importante meseta plástica), como el pandeo plástico característico de barras de reducida esbeltez $y$, finalmente, el pandeo brusco y repentino en las barras de esbeltez intermedia.

La formulación así obtenida, además de la elevada coincidencia con los resultados experimentales de la bibliografía disponible, presenta la ventaja de su gran facilidad para el tratamiento informático y, por tanto, de su utilidad para el análisis de estructuras espaciales complejas.

http://informesdelaconstruccion.revistas.csic.es 


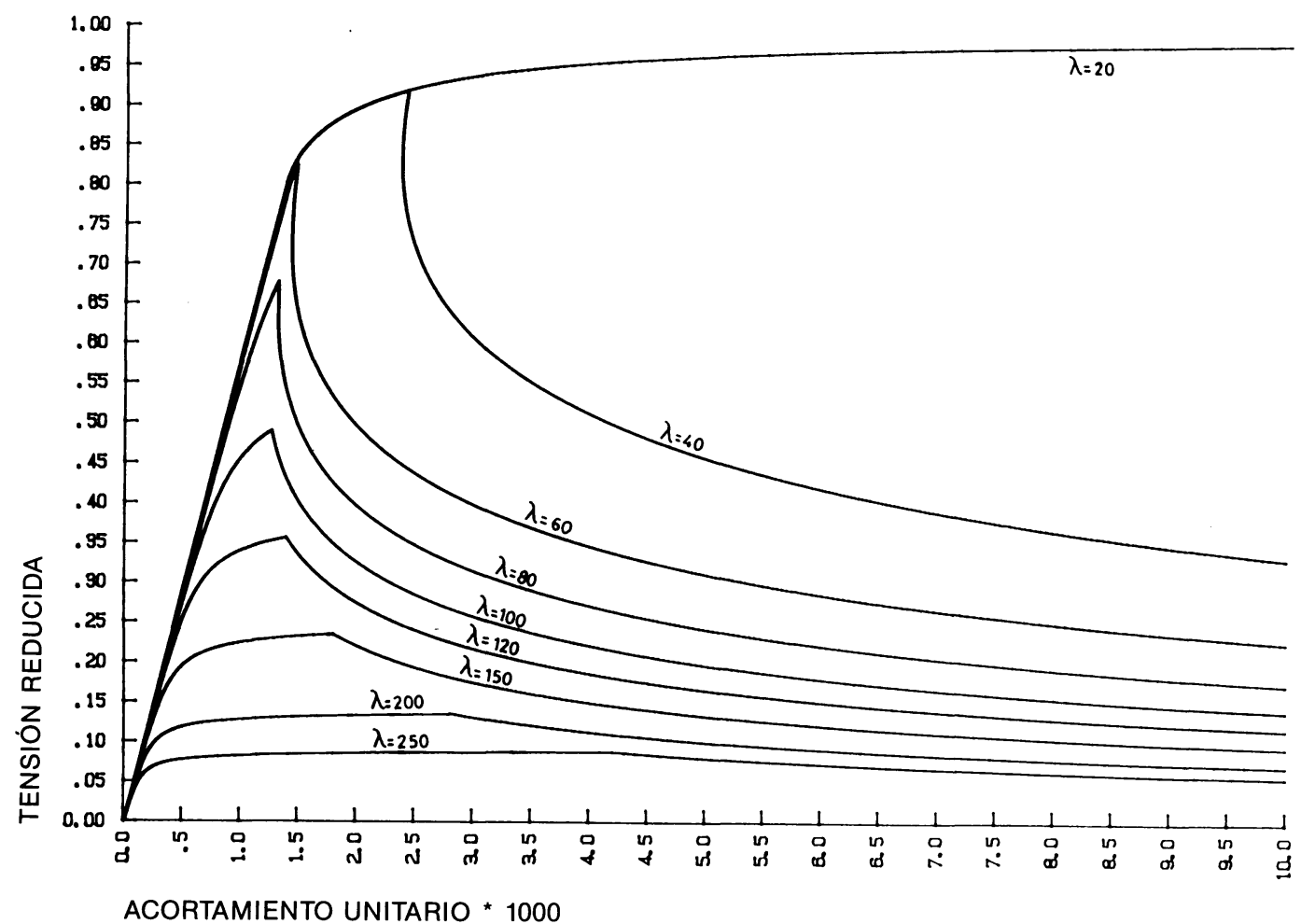

Fig. 7. CURVAS CONSTITUTIVAS DE BARRAS COMPRIMIDAS. Imperfección L/500 Acero A52.

\section{ANÁLISIS NO LINEAL DE MALLAS ESPACIALES}

Para plantearnos el análisis no lineal de las mallas espaciales se precisa, por una parte, la idealización de las relaciones constitutivas de los perfiles tubulares $y$, por otra, el desarrollo de un programa de cálculo que a través de un algoritmo incremental iterativo nos permita describir todo el proceso carga-deformación de la estructura.

Respecto a la idealización del comportamiento de las barras se ha recurrido a la técnica de modificación del módulo de elasticidad prescindiendo de otros procedimientos tales como el de eliminación de la estructura de las barras críticas por cuanto la simplicidad de esta técnica conlleva el prescindir de la colaboración de dichas barras en el comportamiento de la malla.

El procedimiento consiste, entonces, en modificar el módulo de elasticidad de las barras que alcanzan algún estado crítico dentro de su ley tensión-deformación idealizada. Se ha utilizado con dicha técnica el concepto que hemos convenido en llamar de BANDAS CRÍTICAS, de manera que en cada iteración de cálculo se modifica el módulo de todas aquellas barras cu- yos factores de carga crítica no originen variaciones de carga total en la malla superiores a un valor prefijado. Este planteamiento presenta la extraordinaria ventaja de reducir ciclos de cálculo al alcanzar situaciones críticas simultáneamente varias barras de la estructura que conforman la "BANDA CRÍTICA" de esa iteración. Además esta decisión se ha tomado tras comprobar en numerosos ejemplos que no se producían diferencias apreciables en la respuesta de la estructura.

La idealización de la curva según se ve en la Fig. 8 consta de un total de siete tramos que permiten tener en cuenta el comportamiento lineal, las mesetas plásticas de tracción y compresión para enlace con la rama decreciente de postpandeo (por tanto, su longitud depende de la esbeltez de la barra), la rama de endurecimiento por deformación en la zona de tracción y los tramos de descarga del postpandeo hasta alcanzar la "carga remanente" o "residual". Asimismo se han previsto las correspondientes ramas de descarga o recuperación elástica dentro de cada tramo.

En la Fig. 9 podemos ver las idealizaciones de las relaciones constitutivas correspondientes a barras imperfectas de acero A42 constituidas por perfiles tubulares acabados en frío. 

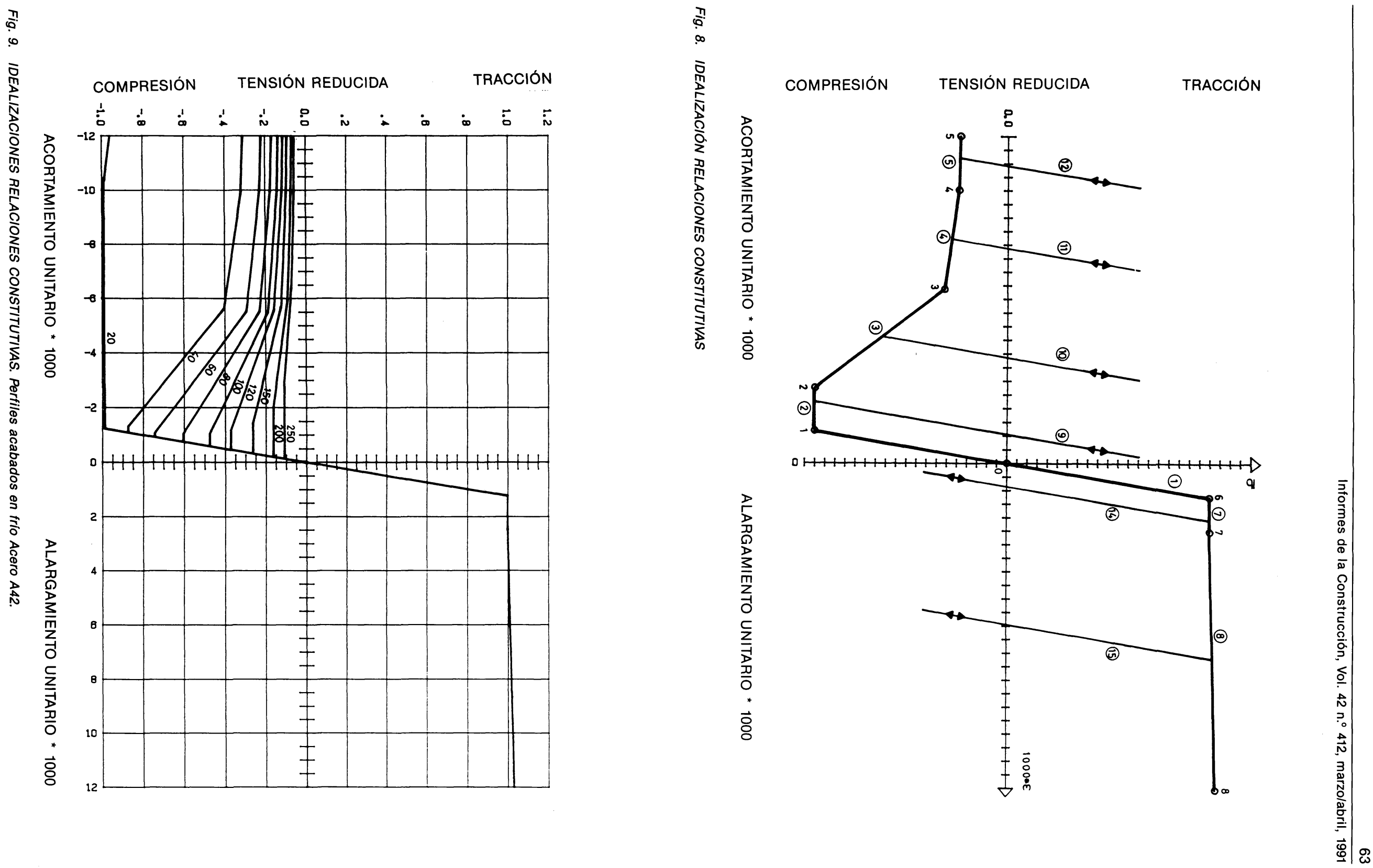
Respecto al problema de la no linealidad geométrica y también con objeto de reducir el número de ciclos de cálculo se procedió a hacer un cálculo lineal hasta el primer estado crítico con lo cual evitamos el proceder por escalones de carga, gracias a la elevada rigidez de las mallas de doble capa. A partir del primer estado crítico y de acuerdo con los resultados obtenidos en los ejemplos analizados, la sucesiva entrada en situaciones críticas de barras de la estructura origina una disminución de rigidez y, en consecuencia, se precisa la consideración del planteamiento del equilibrio sobre la geometría deformada.

\section{PROGRAMAS DE ORDENADOR DESARROLLADOS}

Con objeto de proceder al análisis no lineal de diferentes mallas se precisó la elaboración de tres paquetes de programas que han sido desarrollados en BASIC compilado e instalados en un Tandon AT.

Dichos programas son:
PROGRAMAS DE GENERACIÓN AUTOMÁTICA DE MALLAS. Permiten la generación de 4 tipologías de malla con diferentes condiciones de carga, apoyo y borde.

PROGRAMAS DE ANÁLISIS LINEAL CON DIMENSIO. NADO AUTOMÁTICO DE LAS ESTRUCTURAS. Para el dimensionado se ha acudido al criterio de conseguir mínimo peso aplicando las determinaciones de la MV 103.

PROGRAMAS DE ANÁLISIS NO LINEAL DE MALLAS ESPACIALES. (Fig. 10). Tienen en cuenta los dos tipos de no linealidades geométrica y resistente a las que hemos aludido anteriormente.

En relación con los programas de ordenador desarrollados hay que hacer especial referencia a la vinculación tanto del programa de generación de mallas como el de análisis no lineal con los PROGRAMAS DE DIBUJO Y DISEÑO ASISTIDO a través de ficheros DXF lo que supone una notable aportación tanto para la representación y visualización de la estructura como para ver el proceso de los sucesivos estados críticos de la estructura y los esquemas de rotura de la misma.

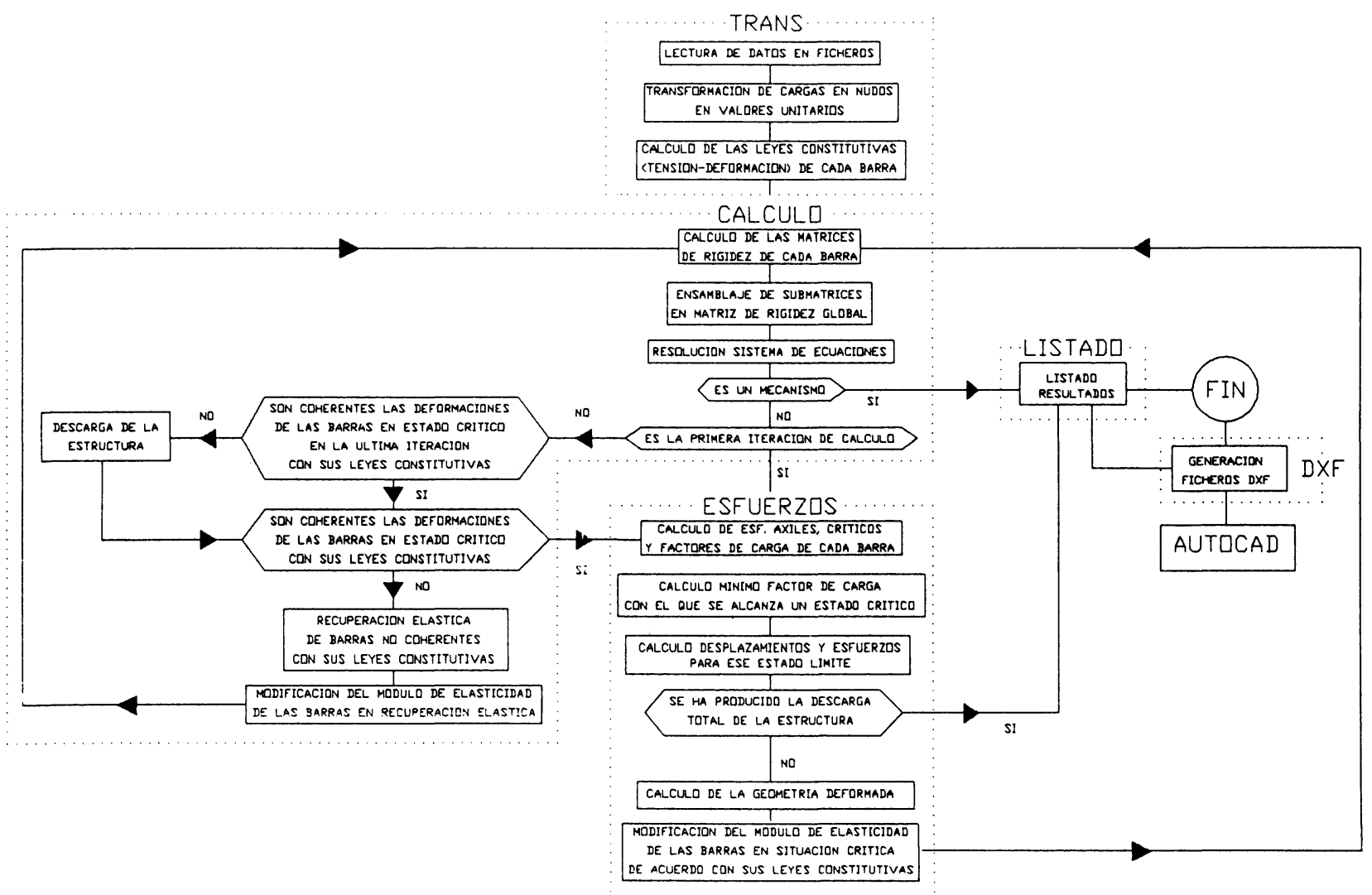

Fig. 10. DIAGRAMA DE FLUJO DE PROGRAMAS DE ANÁLISIS NO LINEAL 


\section{APLICACIONES}

Los programas desarrollados se han aplicado al análisis no lineal de más de 100 estructuras de 4 tipologias donde se han estudiado tanto estructuras planas como cilíndricas y esféricas con diferentes condiciones de borde, apoyo y tipos de barras empleadas, así como distintas idealizaciones del comportamiento no lineal de los perfiles tubulares. En las Figs. 11 a 16 se acompañan tres ejemplos de mallas y sus correspondientes gráficas deformación-carga crítica.

Asimismo, se han realizado estudios comparativos del modelo propuesto con los resultados teóricos y experimentales obtenidos de la bibliografía disponible, que han puesto de manifiesto su validez y utilidad para el análisis no lineal de mallas espaciales.

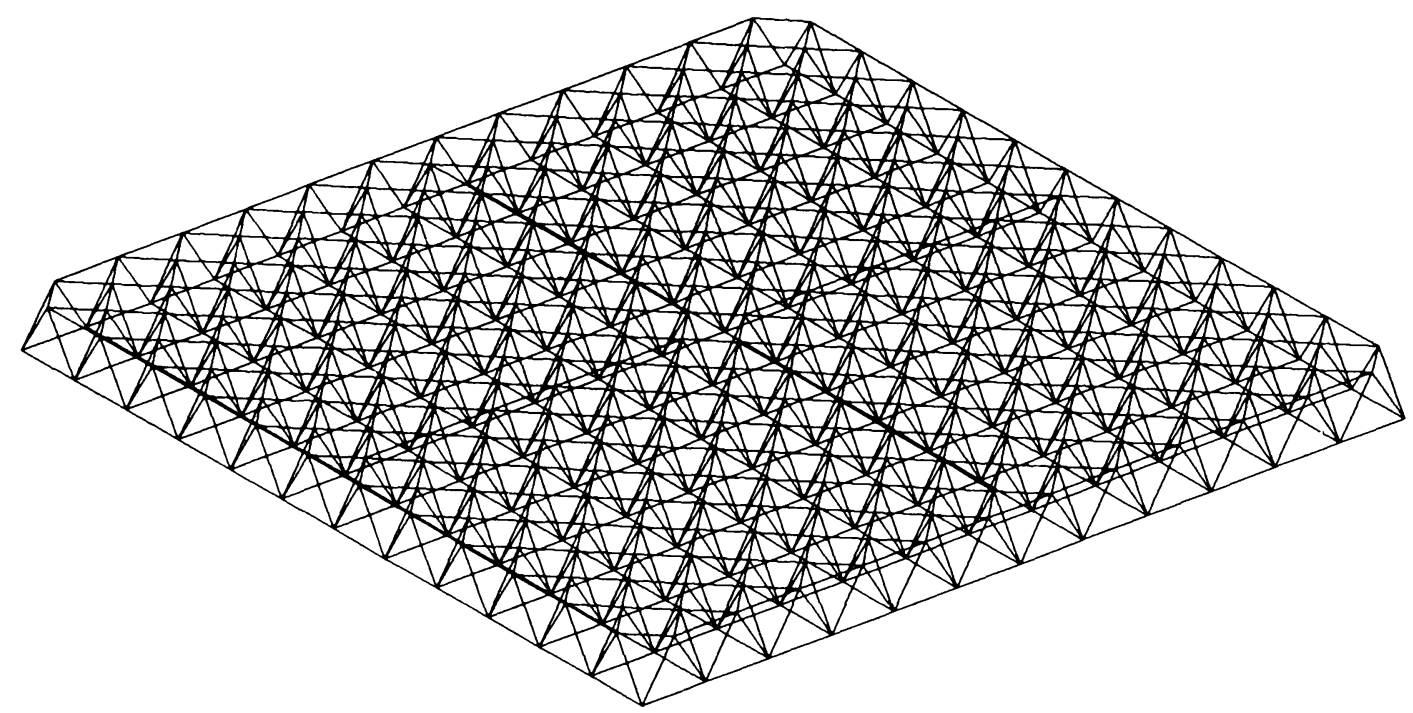

Fig. 11. MALLA ESPACIAL PLANA MÓDULOS PRISMÁTICOS RECTOS

$S=900 \mathrm{~m}^{2} . Q=150 \mathrm{~kg} / \mathrm{m}^{2} \cdot P=10,55 \mathrm{~kg} / \mathrm{m}^{2}$.

Apoyada en ocho soportes.

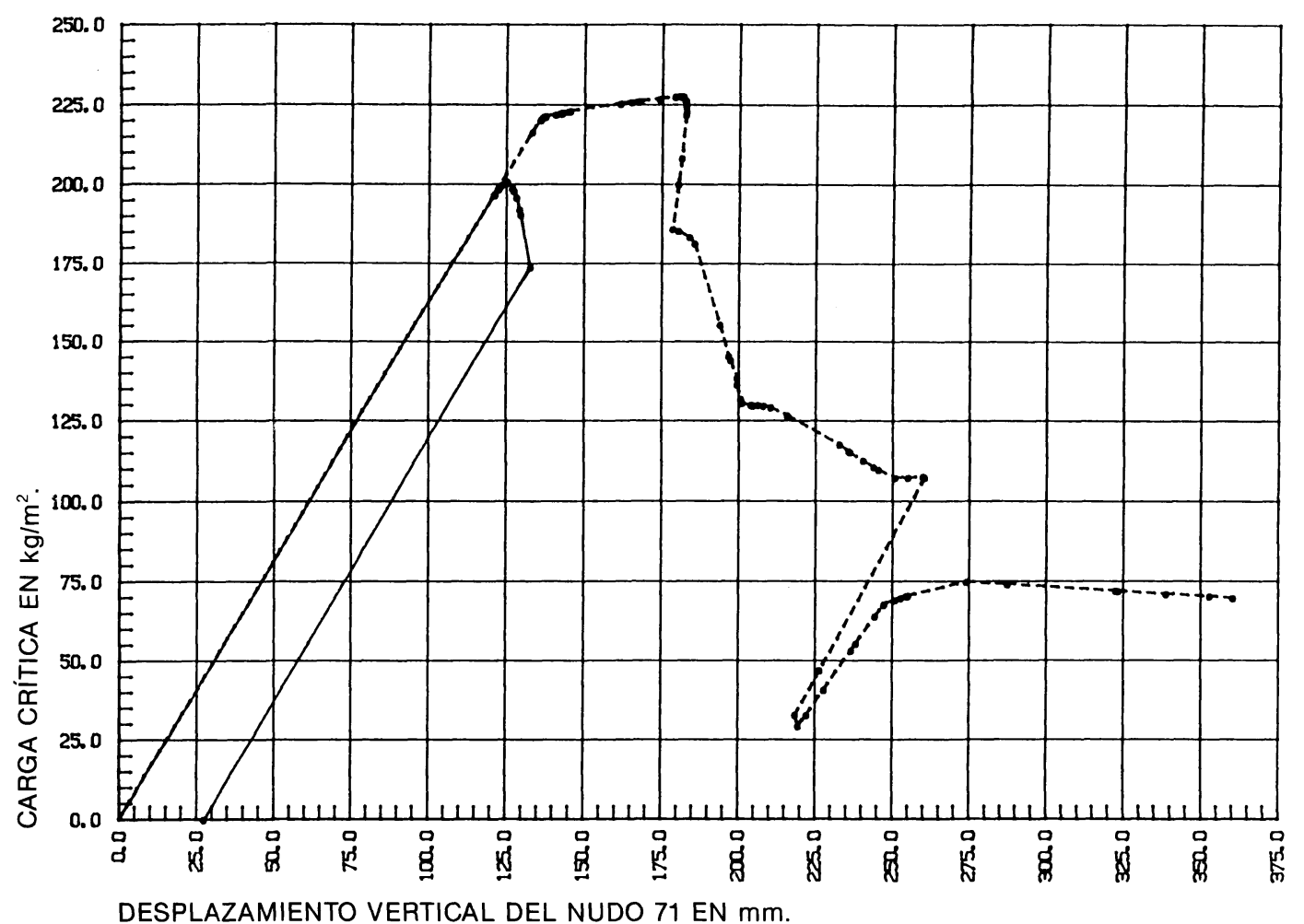

Fig. 12. GRÁFICO DEFORMACIÓN-CARGA CRÍTICA MALLA PLANA 
La aplicación del modelo elaborado a las diferentes estructuras analizadas nos ha permitido obtener interesantes conclusiones respecto al comportamiento de las mismas, entre las que podemos señalas las siguientes:

- Una de las pautas generales que se han observado es que si el dimensionado estricto de la estructura se hace de acuerdo con la normativa de obligado cum- plimiento MV 103, el empleo de perfiles acabados en frío o caliente condiciona el valor de la carga última, la reserva de capacidad de carga y, en definitiva, el trazado de la curva carga-deformación de la malla, como consecuencia de que la referida normativa no distingue entre ambos tipos de perfiles y las cargas críticas de pandeo que proporciona para el dimensionado de valores intermedios a los experimentales correspondientes a los perfiles acabados en frío y caliente.

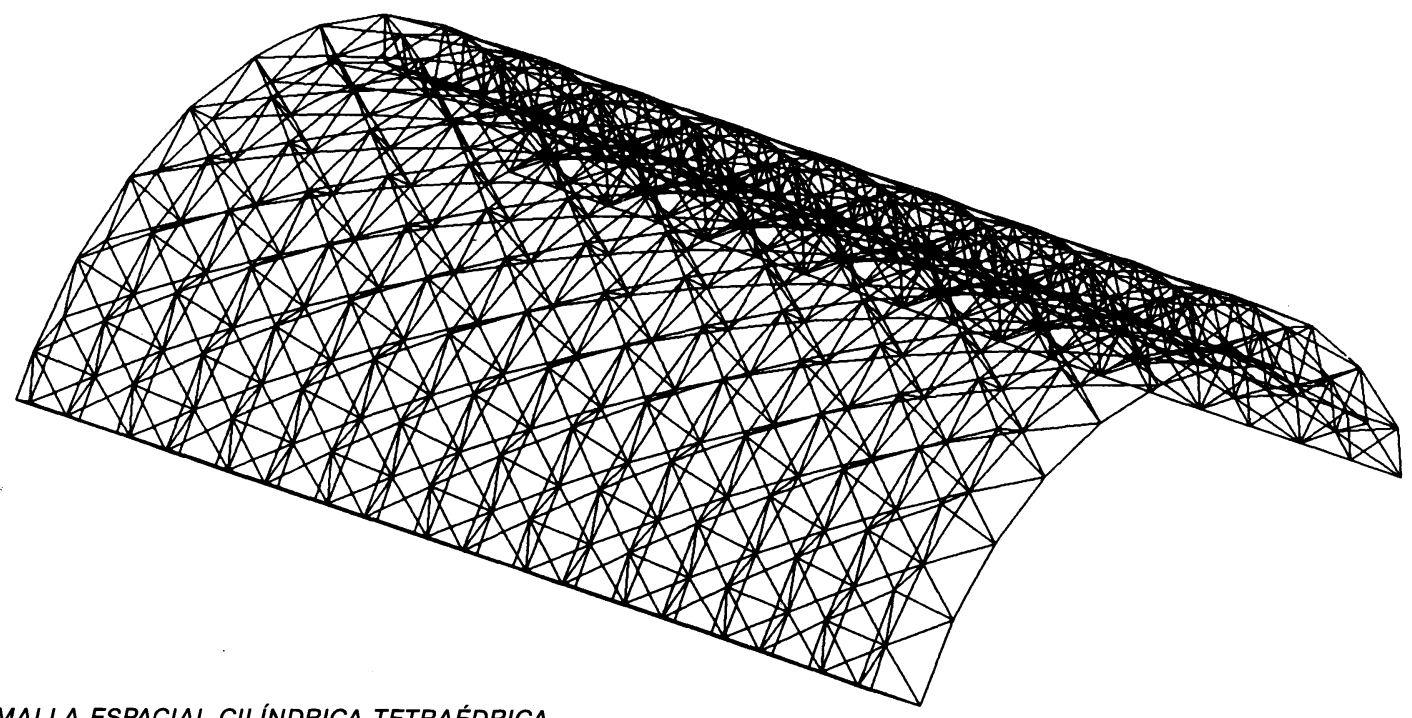

Fig. 13. MALLA ESPACIAL CILÍNDRICA TETRAÉDRICA $S=974 \mathrm{~m}^{2} \cdot Q=150 \mathrm{~kg} / \mathrm{m}^{2} . P=14,77 \mathrm{~kg} / \mathrm{m}^{2}$.

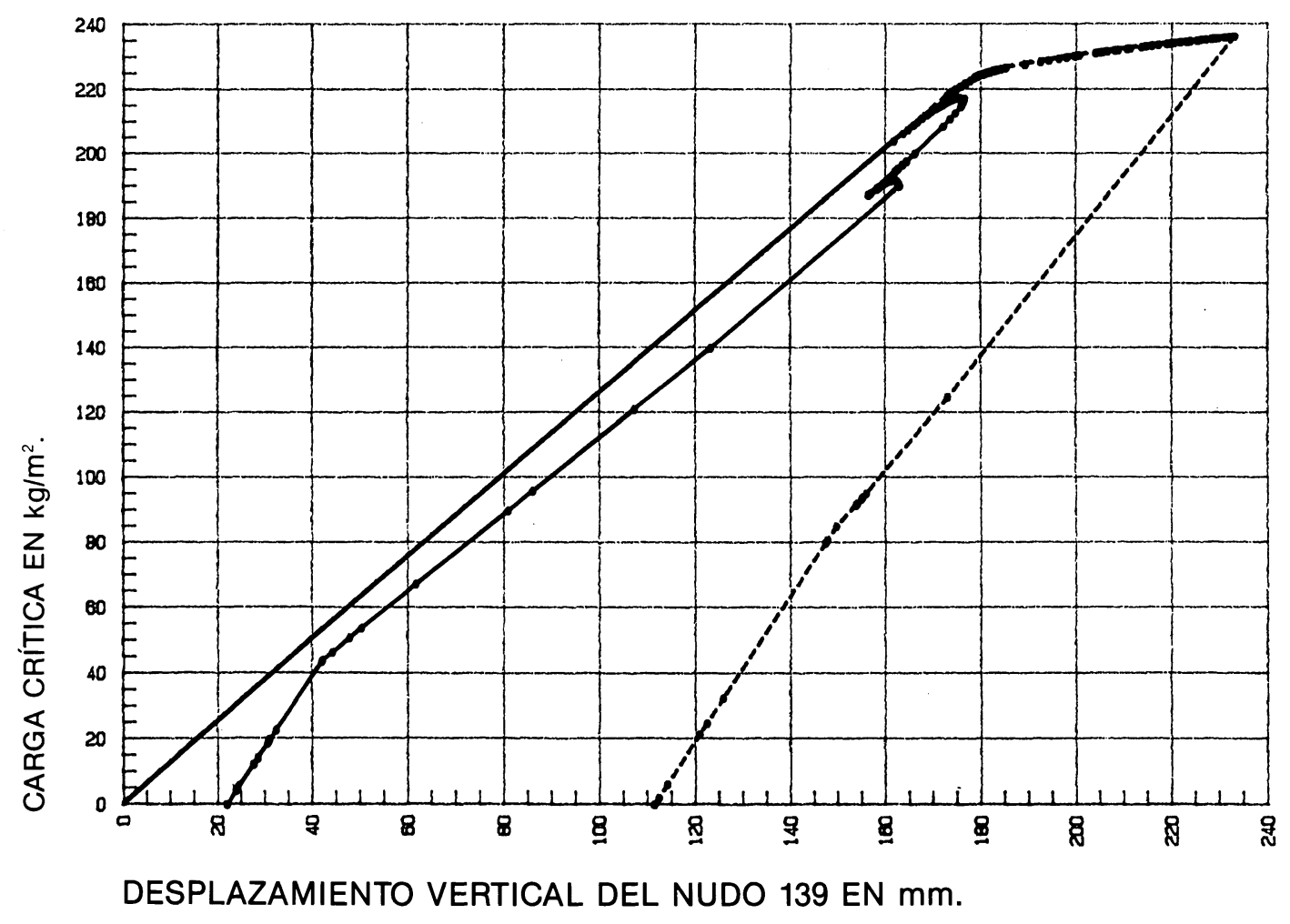

Fig. 14. GRÁFICA DEFORMACIÓN-CARGA CRÍTICA MALLA CILINDRICA.

Perfiles acabados en frio

. Perfiles acabados en caliente ........ 
- Otra conclusión avalada por las aplicaciones realizadas es que el dimensionado estricto de la malla conduce a valores de carga última y curvas carga-deformación radicalmente diferentes de las correspondientes a los dimensionados uniformes. Asimismo, el número de barras que alcanzan situaciones críticas es muy superior a las que se producen cuando el dimensionado es uniforme por capas.
- También es interesante señalar, tal como se ve en las gráficas de las figuras que acompañan al artículo, que las mallas con dimensionado estricto carecen de reserva alguna de capacidad de carga, de manera que después del primer estado crítico no se consigue incremento de carga significativo, contrariamente a las pautas de comportamiento observadas en mallas con dimensionados uniformes.

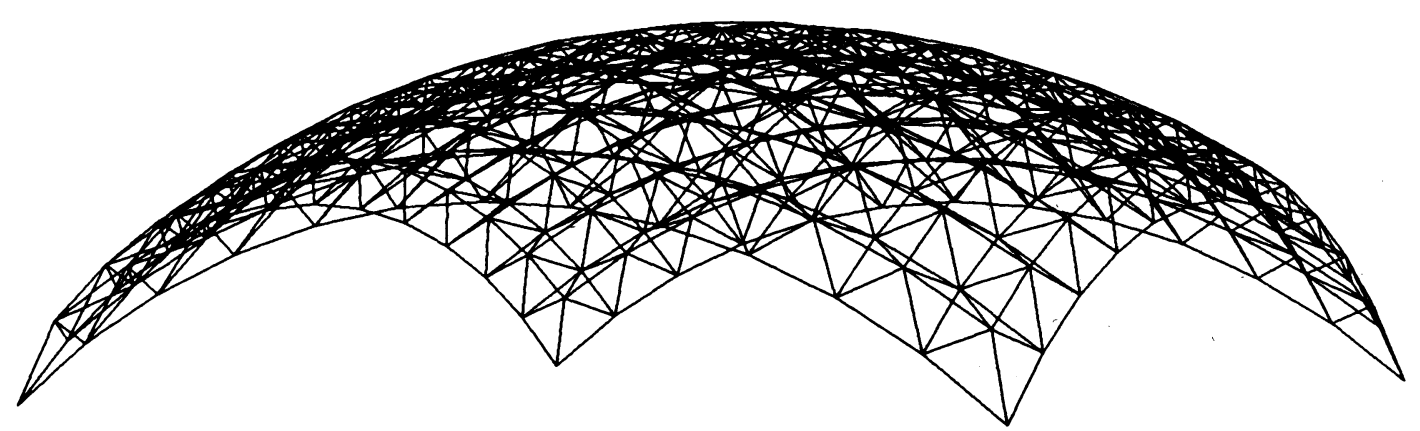

Fig. 15. MALLA ESFÉRICA DE SEMIOCTAEDROS

$S=900 \mathrm{~m}^{2} . Q=100 \mathrm{~kg} / \mathrm{m}^{2} . P=10,81 \mathrm{~kg} / \mathrm{m}^{2}$. Apoyada en cuatro soportes.

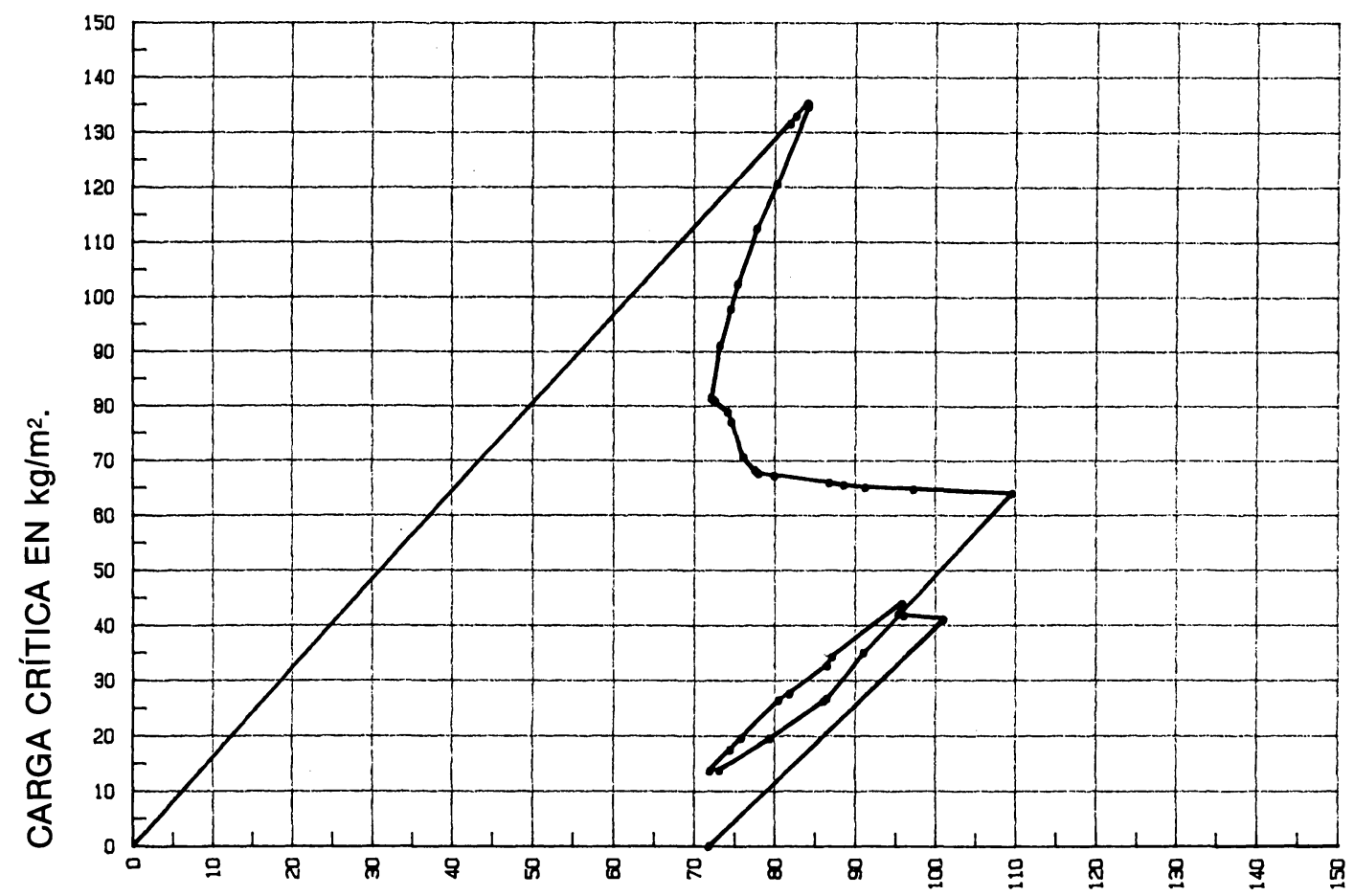

DESPLAZAMIENTO VERTICAL DEL NUDO $50 \mathrm{EN} \mathrm{mm}$.

Fig. 16. GRÁFICA DEFORMACIÓN-CARGA CRÍTICA MALLA ESFÉRICA. Perfiles acabados en frio 
- En general, es preferible, dentro del dimensionado de la estructura, hacer éste de forma que las barras que alcancen estados críticos sean barras traccionadas, pues ello conduce a un comportamiento más plástico, con amplias deformaciones en las que se mantiene la capacidad de carga de la estructura, si bien ello no conlleva, generalmente, un incremento significativo de la carga última.

El modelo de análisis desarrollado nos ha servido también para el estudio y comparación del comportamiento estructural de diferentes criterios de dimensionado para una misma estructura y para proponer un nuevo método de diseño optimizado de estas estructuras basado en la consecución de mínimo peso a partir del análisis no lineal modificando en sucesivas iteraciones las barras que alcanzan los primeros estados críticos y que, por tanto, constituyen los elementos que determinan el máximo valor de carga crítica alcanzable por la estructura. Este método propuesto nos permite una mejora de rendimiento estructural lo que constituye, en mi opinión, un nuevo avance en el aligeramiento de estas estructuras y una mejora de su seguridad.

El estudio de diferentes criterios de dimensionado y las modificaciones efectuadas en las dimensiones de las secciones de algunas barras nos permiten asimismo concluir que la capacidad de las mallas espaciales para "tapar" errores de dimensionado basándose en su capacidad de redistribución de esfuerzos, es muy reducida cuando se emplea el criterio de dimensionado estricto, de manera que pequeñas reducciones de secciones en las barras originan importantes pérdidas de carga última de la estructura y cambios en la forma de la curva carga-deformación. Dicho de otra manera, pequeñas reducciones en las secciones de las barras de una malla con dimensionado estricto, originan significativas disminuciones de su rendimiento estructural.

Los resultados obtenidos con todas las mallas analizadas se han resumido en unos gráficos de barras relativos al rendimiento estructural para diferentes tipologías y diferentes criterios de dimensionado, de los cuales puede verse uno en la Fig. 18 correspondiente a mallas cilindricas.

Por último, la vinculación de los programas de cálculo y diseño asistido se ha mostrado como un instrumento de extraordinaria utilidad y posibilidades de desarrollo, tanto para averiguar formas de rotura, proceso de deformación seguidos por la malla hasta alcanzar el estado límite último o, simplemente, representación gráfica de la estructura.

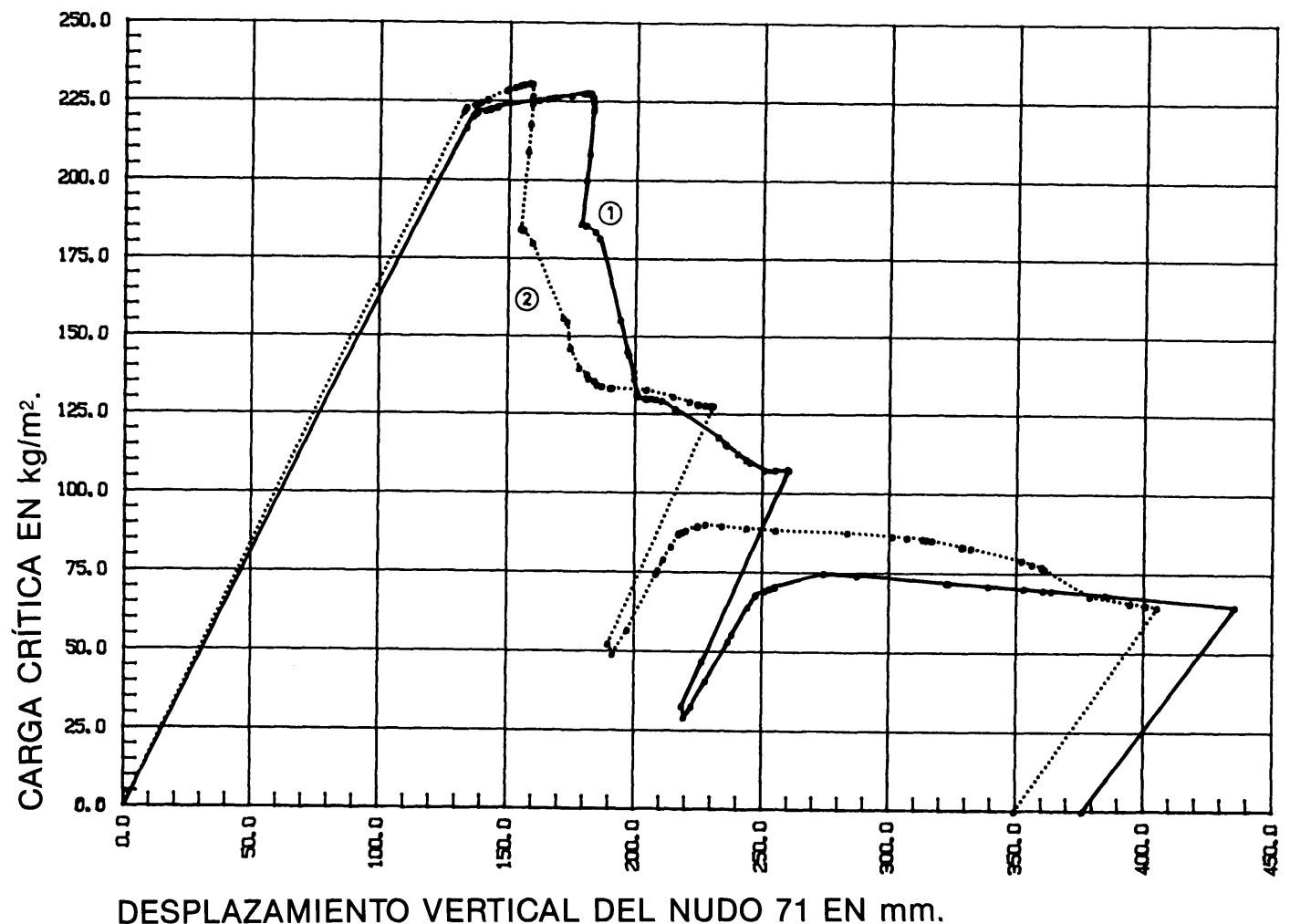

Fig. 17. INFLUENCIA DEL DIMENSIONADO DE BARRAS. 1.-Dimensionado estricto. 2.-Redimensionado con análisis no lineal 


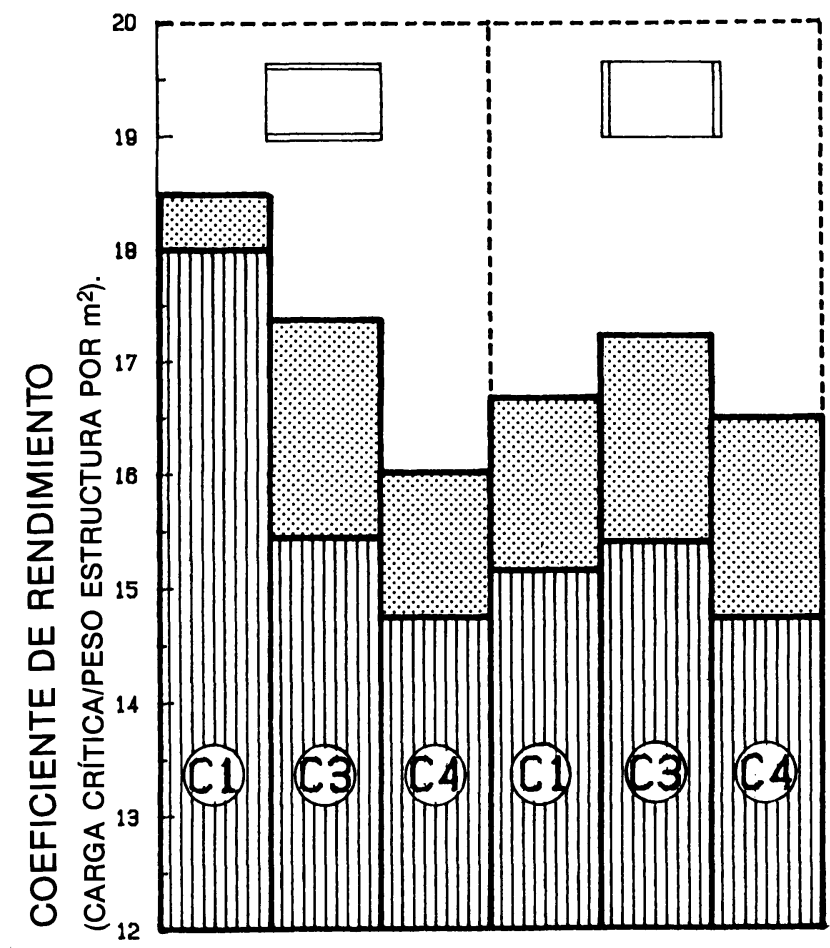

\section{TIPOLOGÍAS DE MALLAS ESPACIALES}

Fig. 18. GRÁFICO RENDIMIENTO ESTRUCTURAL MALLAS CILINN. DRICAS

C1.- Malla de semioctaedros.

C3.-Malla de módulos prismáticos rectos.

C4. - Malla tetraédrica.

\section{CONCLUSIONES}

En definitiva, hemos obtenido la formulación analítica de las leyes constitutivas de perfiles tubulares huecos de sección circular, lo que nos da tanto la carga crítica de pandeo como la descripción completa de todo el proceso seguido por una barra comprimida antes y después de alcanzarse dicha carga crítica.

Con las ecuaciones constitutivas del comportamiento no lineal de barras comprimidas estábamos en condiciones de proponer un modelo de análisis no lineal de mallas espaciales a partir de la linearización de dichas leyes constitutivas y de la aplicación de un modelo incremental iterativo en el que en cada iteración se modifica el módulo de elasticidad de las barras en estado crítico que componen la llamada "BANDA CRíTICA" de esa iteración, teniendo en cuenta, en el planteamiento del equilibrio, la geometría deformada de la estructura.

El modelo de análisis propuesto se aplicó a una amplia variedad de estructuras tanto planas como cilíndricas y esféricas que nos han permitido extraer conclusiones de gran interés tanto relativas al comportamiento de las mismas hasta alcanzarse el estado límite como a las diferencias de comportamiento de la estructura según los criterios de dimensionado empleados, como a los diferentes rendimientos estructurales de las mallas analizadas. Asimismo se propone un nuevo método de diseño basado en el análisis no lineal que conduce a una mejora de rendimiento y por tanto a un mayor aligeramiento estructural.

\section{AGRADECIMIENTOS}

Este trabajo ha sido realizado con financiación proporcionada por la DGICYT a través de un proyecto de investigación en el que participa el autor.

\section{REFERENCIAS BIBLIOGRAFICAS}

ATIENZA, José Ramón / MIRAGALL, Carlos / IRLES, Ramón (1986) "Relaciones constitutivas de los perfiles tubulares de pared delgada utilizados en la construcción de mallas espaciales de nudos articulados". Informes de la Construcción, Vol. 38 núm. 381 Junio.

COLLINS, I. M. (1981) "Collapse analysis of double-layer grids". PhD Thesis University of Surrey.

COLLINS, I. M. I SUPPLE, W. J. (1979) "Experimental post-buckling curves for tubular struts". Space research centre report University of Surrey.

CHA, S. L. I KITIPORNCHAI, S. (1988) "Inelastic postbuckling behaviour of tubular struts". Journal of the structural engineering Vol. 114 Págs. 1.091-1105.

ESTÉVEZ CIMADEVILA, F. J. (1990) "Análisis no lineal de mallas espaciales de doble capa con dimensionado estricto". Tesis Doctoral Universidad de La Coruña.

KITIPORNCHAI, S. I AL-BERMANI, F. G. A. I CHAN, S. L. (1988) "Geometric and material nonlinear analysis of structures comprising rectangular hollow sections". ENGINEERING STRUCTURES Vol. 10 Págs. 13-23.

MEEK, J. L. I LOGANATHA, S. (1989) "Geometric and material nonlinear behaviour of structures comprising circular and rectangular hollow sections". 10 YEARS OF PROGRESS IN SHELL AND SPATIAL STRUCTURES. IASS Madrid 11-15 Septiembre Vol. 4.

SAKA, T. (1989) "Approximate analysis method for postbuckling behaviour of double-layer space grids constructed by a bolted jointing system". 10 YEARS OF PRO. GRESS IN SHELL AND SPATIAL STRUCTURES. IASS Madrid 11-15 Septiembre Vol. 4. 
SUGIMOTO, H. I CHEN, W. F. (1985) "Inelastic postbuckling behaviour of tubular members". JOURNAL OF THE STRUCTURAL ENGINEERING Vol. 111 Págs. 1.9651.978.

SUPPLE, W. J. I COLLINS, I. M. (1980) "Post-critical behaviour of tubular struts". ENGINEERING STRUCTURES.

SUPPLE, W. J. I COLLINS, I. M. (1981) "Limit state analysis of double-layer grids". SPACE RESEARCH CENTRE. DEPARTMENT OF CIVIL ENGINEERING University of Surrey Págs. 93-117

\section{NOTACIÓN EMPLEADA}

\section{Minúsculas romanas}

$a_{0}$.......... flecha inicial de una barra imperfecta

\section{Mayúsculas romanas}

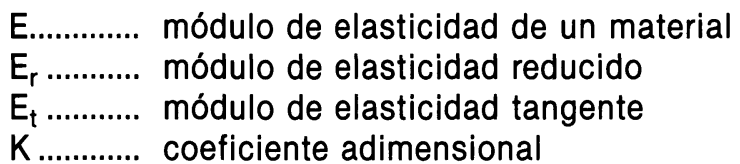

L............ longitud de una barra
$N$.......... fuerza exterior
$\tilde{N}$........... coeficiente de reducción al pandeo
X........... eje coordenado
Y............ eje coordenado
Z............ eje coordenado

\section{Minúsculas griegas}

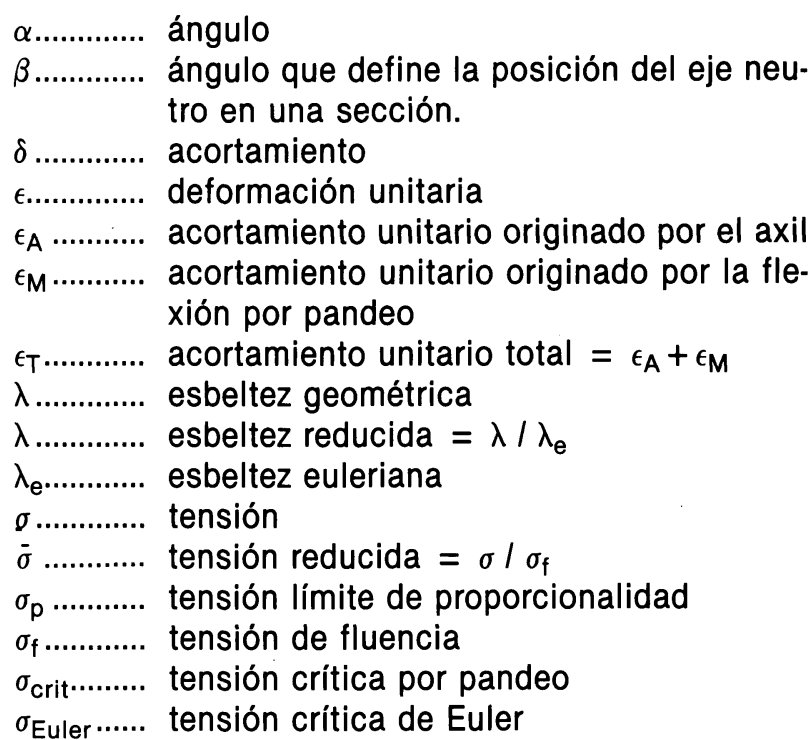

\section{publicación del ICCET/CSIC}

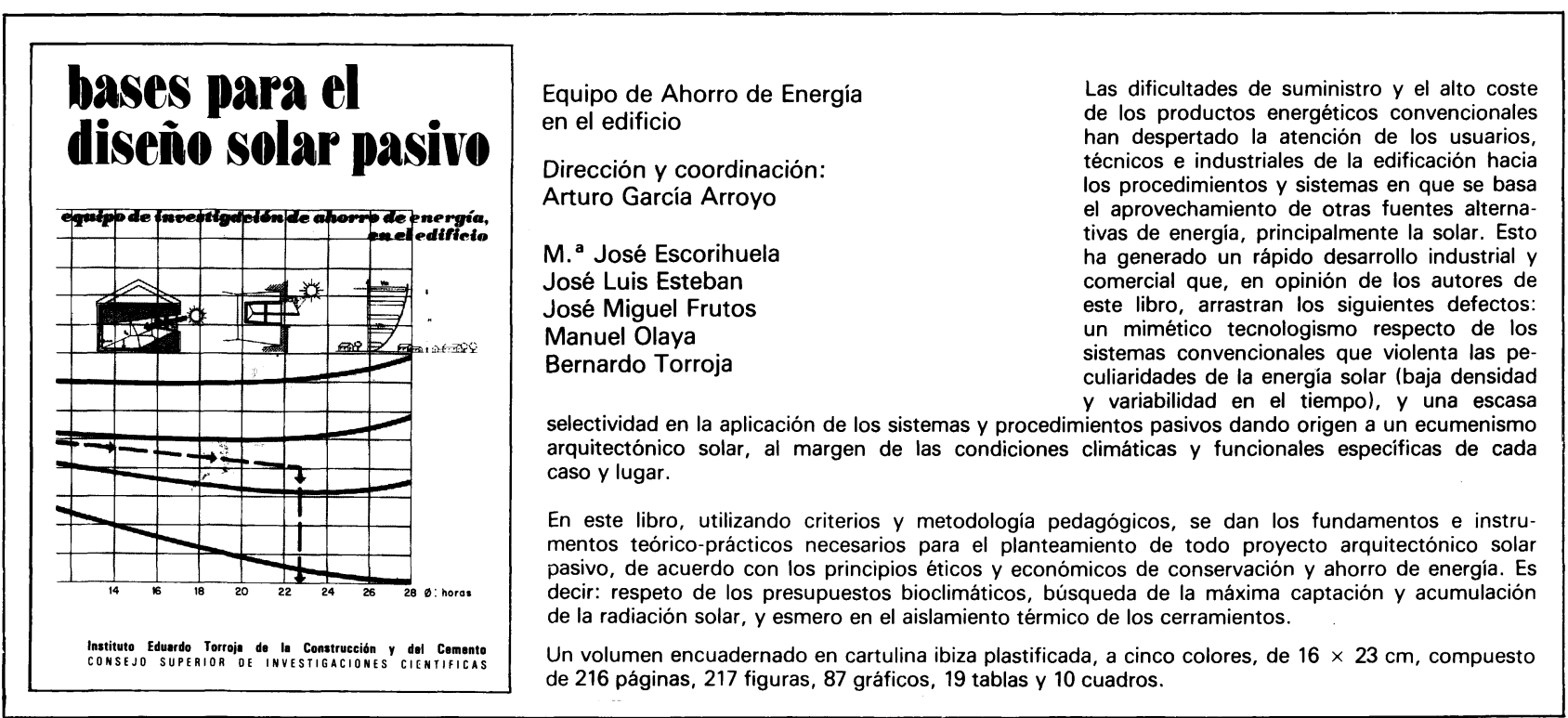

